\title{
A SARS-CoV-2 Coronavirus Antigen-Detecting Half-Strip Lateral Flow Assay Towards the Development of Point of Care Tests Using Commercially Available Reagents
}

\author{
Authors \\ Benjamin D. Grant ${ }^{1}$, Caitlin E. Anderson ${ }^{1}$, John R. Williford ${ }^{1}$, Luis F. Alonzo ${ }^{1}$, Veronika A. Glukhova ${ }^{1}$, David \\ S. Boyle ${ }^{2}$, Bernhard H. Weigl ${ }^{1}$, Kevin P. Nichols ${ }^{1 *}$ \\ 1 - Intellectual Ventures Lab, Bellevue Washington, USA \\ 2 - PATH, Seattle Washington, USA \\ * Corresponding author: knichols@intven.com
}

\begin{abstract}
$\underline{\text { Abstract }}$
The SARS-CoV-2 pandemic has created an unprecedented need for rapid diagnostic testing to enable the efficient treatment and mitigation of COVID-19. The primary diagnostic tool currently employed is reverse transcription polymerase chain reaction (RT-PCR), which can have good sensitivity and excellent specificity. Unfortunately, implementation costs and logistical problems with reagents during the global SARS-CoV-2 pandemic have hindered its universal on demand adoption. Lateral flow assays (LFAs) represent a class of diagnostic that, if sufficiently clinically sensitive, may fill many of the gaps in the current RT-PCR testing regime, especially in low- and middle-income countries (LMICs). To date, many serology LFAs have been developed, though none meet the performance requirements necessary for diagnostic use cases, primarily due to the relatively long delay between infection and seroconversion. However, based on previously reported results from SARS-CoV-1, antigen-based SARS-CoV-2 assays may have significantly better clinical sensitivity than serology assays. To date, only a very small number of antigen-detecting LFAs have been developed. Development of a half-strip LFA is a useful first step in the development of any LFA format. In this paper we present a half-strip LFA using commercially available antibodies for the detection of SARS-CoV-2. We have tested this LFA in buffer and measured an LOD of $0.62 \mathrm{ng} / \mathrm{mL}$ using an optical reader with sensitivity equivalent to a visual read. Further development, including evaluating the appropriate sample matrix, will be required for this assay approach to be made useful in a point of care setting, though this half-strip LFA may serve as a useful starting point for others developing similar tests.
\end{abstract}

\section{Introduction}

In late 2019, a novel coronavirus, SARS-CoV-2 was identified in China with significant mortality, morbidity, and infectiousness. ${ }^{1}$ By January 2020, SARS-CoV-2 had spread outside China, including to the United States. ${ }^{2}$

Rapid testing for SARS-CoV-2, the virus which causes COVID-19, is urgently needed early in the onset of the disease to effectively control the spread of SARS-CoV-2 within a population. ${ }^{3,4}$ Both reverse transcription polymerase chain reaction $(\mathrm{RT}-\mathrm{PCR})^{5-7}$ and direct viral antigen testing ${ }^{8,9}$ have the potential 
for diagnosis early in the course of COVID-19, unlike serology assays. ${ }^{10}$ However, RT-PCR is relatively expensive, and the supply chain required to effectively conduct a population-scale case finding activity using RT-PCR has been severely strained by the SARS-CoV-2 pandemic. ${ }^{5}$ Inadequate availility of RT-PCR testing capacity has hindered response efforts even in well-funded health care systems. ${ }^{11}$ In low and middle income countries, the situation is even more dire, with testing rates currently orders of magnitude below that of high income countries.

Lateral flow assay (LFA) antigen tests may be an inexpensive, scalable solution to population scale diagnosis of SARS-CoV-2 in both high-income countries and LMICs. LFAs have been shown to be a scalable, easily mass producible test platform, with on the order of a half billion LFAs for malaria alone being sold each year, many for approximately USD $\$ 0.25 .^{12}$

Compared to serology tests - which detect the presence of a target antibody - antigen based LFAs are less sensitive than RT-PCR, but may approach the clinical sensitivity of RT-PCR with further research and development. For example, Standard Diagnostics recently commercialized a visually read antigen detecting LFA (catalog number 09COV30D) with a self-reported overall sensitivity of $84 \%$, and specificity of $100 \%$ compared to RT-PCR. ${ }^{13}$ While this is an excellent start, further research is needed to improve upon this sensitivity. It has been postulated that higher viral load may be associated with more severe outcomes and therefore an LFA that can rapidly detect high viremia may have a role in identifying those most at risk of poor outcomes. ${ }^{14}$

Many LFAs for SARS-CoV-2 are in development, though the vast majority of these are serological tests for previous exposure to SARS-CoV-2. As of May $1^{\text {st }}, 2020$, over 300 immunoassays had been reported to the Foundation for Innovative and New Diagnostics (FIND) as being in development to detect SARS-CoV- 2 . However, $<20$ of these 300 appear to be intended to detect antigens, with the naming of the remaining immunoassays implying they are serology assays. ${ }^{15}$ Thus it appears the state of development of antigen based assays significantly lags behind that of serology tests for SARS-CoV-2.

SARS-CoV-2 serology assays of all formats, including LFAs, may be useful for both epidemiological purposes and to act as an "immunity passport" in populations with a sufficiently high prevalence to allow for acceptable positive predictive values. ${ }^{16-19}$ However, the inability to detect the early onset of COVID-19 means serology LFAs are not considered useful for case detection or diagnosis of SARS-CoV-2 infection, for the purposes of treatment or isolation. For example, in a recent study of the VivaDiag COVID-19 IgM/IgG rapid test, a serologic LFA, the clinical sensitivity was only $18.4 \%$ vs RT PCR, with a specificity of $91.7 \%{ }^{10}$

Antigen detecting ELISAs were previously developed in 2004 for SARS-CoV-1, with limits of detection of approximately $50 \mathrm{pg} / \mathrm{mL}$ and clinical sensitivity as a function of days since onset that was significantly better than the useful time window for the current generation of SARS-CoV-2 serology assays. These results further imply there is potential for antigen based assays for the detection of SARS-CoV-2. ${ }^{8,9}$

In this paper we describe a half-strip LFA, which is a test format frequently utilized as the first step in assay development for a "full" LFA. ${ }^{20} \mathrm{~A}$ half-strip (also sometimes referred to as a dipstick) LFA has only a nitrocellulose analytical membrane and a wick pad, without sample or conjugate pads. Sample and conjugate are premixed in a container, such as a 96-well plate, prior to the insertion of the half-strip. The assay described in this paper potentially has utility on its own. However, the assay is primarily intended 
as a launching point to expedite development for others interested in developing an antigen detecting LFA for SARS-CoV-2.

\section{Materials and Methods}

\section{Casein block preparation}

For the preparation of the blocking solution, $6 \mathrm{~g}$ casein was dissolved in $80 \mathrm{~mL}$ of $50 \mathrm{mM} \mathrm{NaOH}$ overnight until fully dissolved. The following day, 0.24 boric acid (B0252, Sigma Aldrich, St. Louis MO, USA) and 0.45 g sodium tetraborate (B9876, Sigma Aldrich,) were added and the $\mathrm{pH}$ was adjusted to $\mathrm{pH} 8.5$. Finally, UltraPure distilled water (10977-015, Invitrogen, Carlsbad, CA) was added to a total volume of $100 \mathrm{~mL}$, yielding $6 \%$ casein in $50 \mathrm{mM}$ borate. The solution was heated to $37^{\circ} \mathrm{C}$ for 7 days and stored at $-20^{\circ} \mathrm{C}$.

\section{Latex bead conjugation}

For the test line conjugate, $400 \mathrm{~nm}$ carboxylic red latex beads (CAR400NM, Magsphere, Pasadena CA, USA) were conjugated to Rockland 200-401-A50 polyclonal antibodies (Rockland Immunochemicals, Inc., Gilbertsville PA) at a weight:weight ratio of 20:1 (beads:antibody) using EDC/NHS coupling for the test line conjugate. For the control line conjugate, $400 \mathrm{~nm}$ carboxylic blue latex beads (CAB400NM , Magsphere) were conjugated to Chicken IgY (ChromPure 003-000-003, Jackson ImmunoResearch, West Grove PA) at a weight:weight ratio of $10: 1$ (beads:antibody) using EDC/NHS coupling.

Stock latex particles were washed, activated using fresh stocks of EDC and NHS diluted in MES buffer, pH6. Latex particles were then conjugated to their respective antibodies, as described above, for $3 \mathrm{hrs}$ on an orbital shaker at room temperature. The conjugates were then quenched overnight using ethanolamine, again on the orbital shaker. Conjugates were then washed and stored in $50 \mathrm{mM}$ borate with 1\% 7-day aged casein, as described under casein block preparation. Concentrations for the completed conjugates were determined by measuring the absorbance at $560 \mathrm{~nm}$ for red and $660 \mathrm{~nm}$ for blue. Final stocks were stored at $4^{\circ} \mathrm{C}$ until use.

\section{Antibody biotinylation}

The antibody was first buffer exchanged into PBS to remove sodium azide using Amicon filters (50 kDA MWCO, UFC5050 Sigma). Specifically, the antibody was concentrated 20-fold and brought back to the original volume with PBS. This was done three times to remove sodium azide. The antibody was biotinylated at $1 \mathrm{mg} / \mathrm{mL}$ with 50 molar excess NHS-dPEG 12 -biotin (10198, Quanta Biodesign, Plain City, OH, USA). Excess biotin was removed using Amicon filters again, this time with five total concentration cycles. The biotinylation ratio was determined to be 7.1 moles of biotin per mole of antibody using the QuantTag Biotin Quantification Kit (BDK-2000, Vector Laboratories, UK).

\section{Nitrocellulose striping}

$20 \mathrm{~mm}$ CN95 was striped with a test line at $8 \mathrm{~mm}$ from the edge of nitrocellulose (upstream from the flow direction) and $13 \mathrm{~mm}$ from upstream edge of nitrocellulose. The test line was striped at $1 \mathrm{mg} / \mathrm{mL}$ polystreptavidin (Cat \#10 120 050, Biotez, Berlin, DE). The control line was striped at $0.5 \mathrm{mg} / \mathrm{mL}$ goat antiChicken IgY (Cat \#703-005-155, Jackson ImmunoResearch) 


\section{Nitrocellulose blocking}

Nitrocellulose strips were blocked in a solution containing $2 \%$ beta-lactose (Sigma, L3750), 0.5\% bovine serum albumin (BSA) (1900-0002, SeraCare, Milford, MA USA), $0.2 \mu \mathrm{m}$ filtered $0.05 \%$ N-lauryl Sarcosine Sodium Salt (LS777, Sigma), 0.3\% 7-day aged casein (prepared as described in method above), in $20 \mathrm{mM}$ AMP at pH 9.0 (A65182, Sigma). Striped nitrocellulose was dipped into blocking buffer, allowing the blocking buffer to wick the nitrocellulose strip until fully wet. Nitrocellulose was then submerged into the blocking buffer for 30 minutes while rocking, after which it was removed and dried flat for $\mathbf{3 0}$ minutes at $25^{\circ} \mathrm{C}$.

\section{LFA assembly}

Blocked nitrocellulose was placed on backing card (MIBA-020, DCN, USA). 20 mm Ahlstrom 320 (AhlstromMunksjo Oyj, Finland) was placed on top of the nitrocellulose with a $3 \mathrm{~mm}$ overlap between the two materials. Excess backing card was cut off. Strips were cut to a $4 \mathrm{~mm}$ width using a Kinematic Matrix guillotine cutter (Kinematic Automaticion, Inc., Twain Harte, CA, USA).

\section{LFA running protocol}

To make $1 \%$ casein in $50 \mathrm{mM}$ borate, $6 \%$ casein (aged 7 days, as prepared above), was diluted in $50 \mathrm{mM}$ borate. Nucleocapsid protein samples (Genscript Cat \#Z03488 and Genemedi GMP-V-2019nCoV-N002) were diluted in PBS (Gibco 10010-023) with 0.05\% tween-20 (SeraCare 5460-0019). Control protein and nucleocapsid antibody conjugated latex stocks were sonicated (Qsonica Model CL-188) with 30 pulses at $25 \%$ power, one second on, one second off, immediately prior to dilution. Latex master mix was prepared at a concentration of $0.0645 \%$ test-line red latex and $0.0011 \%$ control-line blue latex in $50 \mathrm{mM}$ borate and $0.5 \%$ casein. Biotinylated antibody was diluted to $75 \mu \mathrm{g} / \mathrm{mL}$ in PBS with $0.05 \%$ tween-20. Immediately prior to running the LFA, $1 \mu \mathrm{L}$ of biotinylated antibody and $5 \mu \mathrm{L}$ of latex master mix were added to $69 \mu \mathrm{L}$ of sample and mixed by pipetting. The combined $75 \mu \mathrm{L}$ was added to a 96-well plate and an LFA was inserted. After 20 minutes, the LFA was removed and read using an optical LFA reader to construct a semiquantitative calibration curve (AX-2X-S, Axxin, Australia). The Axxin reader does not typically improve the limit of detection (LOD) of the assay compared with the LOD by visual read and was used solely for accuracy in LOD determination.

\section{Limit of detection calculation}

The data was fitted using a four-parameter logistic fit using the drc (Analysis of Dose-Response Curves) package in $\mathrm{R}^{21}$ The fit was weighted inversely to the square root of the signal. The test-line LFA reader score corresponding to the limit-of-detection was defined as

$L O D_{L F A \text { Reader Score }}=\mu_{0}+1.645 \sigma_{0}+1.645 \sigma_{\text {low positive }}$

where $\mu_{0}$ is the mean LFA reader score for the negative samples, $\sigma_{0}$ is the corresponding standard deviations, and $\sigma_{\text {low positive }}$ is the pooled standard deviation for the four lowest non-zero concentrations $(0.25,0.5,1,2 \mathrm{ng} / \mathrm{mL}){ }^{22}$ The corresponding concentration and associated $95 \%$ confidence intervals were then calculated using the fitted curve. 


\section{Results and Discussion}

A dose response curve was generated for the half-strip LFA using two commercially available SARS-CoV-2 nucleocapsid (N) proteins, from Genemedi and Genscript. The limit of detection for the Genemedi $\mathrm{N}$ protein was $0.65 \mathrm{ng} / \mathrm{mL}(95 \% \mathrm{Cl}$ of 0.53 to $0.77 \mathrm{ng} / \mathrm{mL})$ and for the Genscript N protein was $3.03 \mathrm{ng} / \mathrm{mL}$ ( $95 \% \mathrm{Cl}$ of 0.00 to $7.44 \mathrm{ng} / \mathrm{mL}$ ). The dose response curve, with $95 \% \mathrm{Cl}$ calculated in $\mathrm{R}$ using the drc package, is shown in Figure 1.

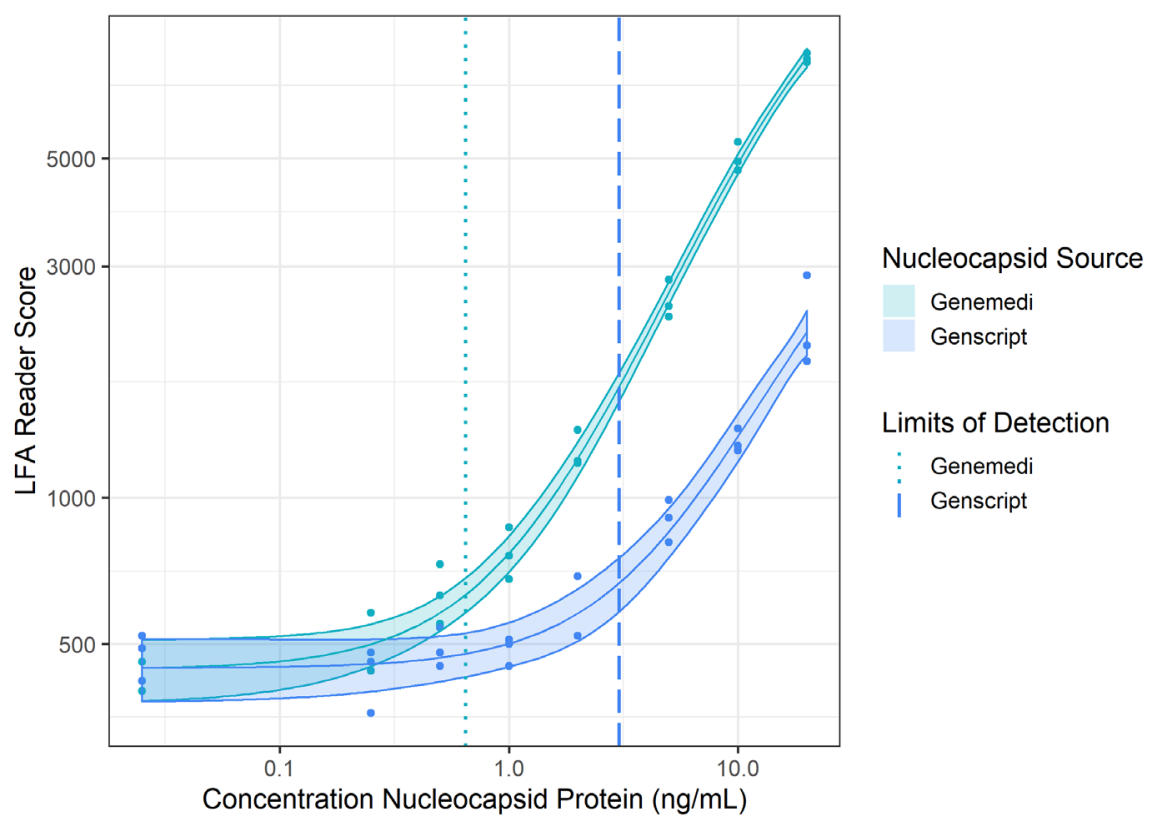

Figure 1 - The dose response curve for a half-strip LFA using nucleocapsid protein from two commercially available sources, as measured using a commercially available optical LFA reader. The LOD for the Genemedi N protein was $0.65 \mathrm{ng} / \mathrm{mL}(95 \% \mathrm{Cl}$ of 0.53 to $0.77 \mathrm{ng} / \mathrm{mL})$ and for the Genscript N protein was $3.03 \mathrm{ng} / \mathrm{mL}(95 \% \mathrm{Cl}$ of 0.00 to $7.44 \mathrm{ng} / \mathrm{mL})$. Raw data for this graph is presented in supplemental information.

It has not yet been determined what analytical sensitivity, in either blood or nasal samples, will be required to meet an acceptable level of clinical sensitivity for SARS-CoV-2. Interestingly, a detection limit of $50 \mathrm{pg} / \mathrm{mL}$ in an ELISA system was shown in 2004 for SARS-CoV-1 to permit positive rates of $\mathrm{N}$ protein detection in sera collected at 1 to 5,6 to 10,11 to 15 , and 16 to 20 days after the onset of symptoms for 414 samples from 298 serologically confirmed patients pf $92.9,69.8,36.4$, and $21.1 \%$, respectively. 8,9

The most significant work that remains for this assay to be usable as a point of care test, if the LOD is near clinically useful ranges, will be the addition of a sample and conjugate pad directly on the strip. Importantly, the best sample to utilize in a point of care device has not yet been determined. Early drafts of target product profiles from WHO and others for an antigen based LFA have thus far specifically called for nasal swab as the sample matrix. Other swab modalities are currently being considered for RT-PCR. ${ }^{23}$ And, previous work on SARS-CoV-1 from 2004 and 2005 in serum showed promising results in an ELISA format. ${ }^{8,9}$ It is our intent that duplication of this work, and determination of realistic limits of detection for a full strip LFA in multiple sample matrices will help point the way towards the best approach for an 
antigen detecting LFA for SARS-CoV-2. Potential sample matrices include extracts from anterior nasal swabs, nasopharyngeal swabs, blood, and saliva, as well as more speculative sample types for point of care tests such as stool. Further, the best antigen target for an antigen detecting SARS-CoV-2 LFA has not been determined, though initial work appears to be focusing primarily on nucleocapsid $(\mathrm{N})$ protein. However, future work should be conducted on feasible clinical sensitivities of spike (S) and membrane (M) proteins of SARS-CoV-2, based on previously reported abundances of these proteins in SARS-CoV- $1 .{ }^{24}$ For the direct immuno-detection of intact SARS-CoV-2 virions, there may also be utility in targeting one antigen for efficient capture and a different antigen for the most sensitive detection.

While the antibodies utilized in this half-strip assay were originally developed for SARS-CoV-1, specificity failures due to cross-reactivity with SARS-CoV-1 are not considered a significant concern for the current SARS-CoV-2 pandemic. While these antibodies have not been used in a published LFA, and significant validation work is still required for any diagnostic application, they have been utilized in several studies specifically investigating SARS-CoV-2. ${ }^{25-27}$ Importantly, the antibodies utilized in this assay are polyclonal antibodies, so particular care must be taken in further studies to rule out cross-contamination with other widely circulating coronaviruses and viruses with similar nucleocapsid proteins. Additionally, application in commercial assays, and any other related polyclonal antibody, must be carefully considered. Other antibodies specific to SARS-CoV-2, some monoclonal, have also been developed, and further screening of additional antibodies will likely improve assay performance.

\section{Conclusion}

In this paper we present a half-strip LFA for the detection of nucleocapsid protein of SARS-CoV-2. This effort is a first step in the development of a functional LFA with the potential for manufacturing at scale. We utilize only commercially available reagents, and conventional protocols, to allow the straightforward duplication and modification of this approach. Further work is required for the development of a practical point of care test for CARS-CoV-2. Further work includes determination of the target antigen(s) that offers greatest sensitivity, screening novel antigen specific antibodies in capture and detector pairs, determination of the optimal sample matrix, and incorporation of the necessary sample preparation into a practical workflow.

\section{Acknowledgements}

Funding provided by The Global Good Fund I, LLC (www.globalgood.com). We thank Puneet Dewan for helpful discussions on the manuscript and clinical needs. 


\section{References}

(1) Zhu, N.; Zhang, D.; Wang, W.; Li, X.; Yang, B.; Song, J.; Zhao, X.; Huang, B.; Shi, W.; Lu, R.; Niu, P.; Zhan, F.; Ma, X.; Wang, D.; Xu, W.; Wu, G.; Gao, G. F.; Tan, W. A Novel Coronavirus from Patients with Pneumonia in China, 2019. N. Engl. J. Med. 2020, 382 (8), 727-733. https://doi.org/10.1056/NEJMoa2001017.

(2) Holshue, M. L.; DeBolt, C.; Lindquist, S.; Lofy, K. H.; Wiesman, J.; Bruce, H.; Spitters, C.; Ericson, K.; Wilkerson, S.; Tural, A.; Diaz, G.; Cohn, A.; Fox, L.; Patel, A.; Gerber, S. I.; Kim, L.; Tong, S.; Lu, X.; Lindstrom, S.; Pallansch, M. A.; Weldon, W. C.; Biggs, H. M.; Uyeki, T. M.; Pillai, S. K. First Case of 2019 Novel Coronavirus in the United States. N. Engl. J. Med. 2020, 382 (10), 929-936. https://doi.org/10.1056/NEJMoa2001191.

(3) Rahimi, F.; Bezmin Abadi, A. T. Case-Finding: Fast, Available, and Efficient Font-Line Diagnostics for SARS-CoV-2. Arch. Med. Res. 2020. https://doi.org/10.1016/j.arcmed.2020.04.008.

(4) Wang, C. J.; Ng, C. Y.; Brook, R. H. Response to COVID-19 in Taiwan: Big Data Analytics, New Technology, and Proactive Testing. JAMA - Journal of the American Medical Association. American Medical Association April 14, 2020. https://doi.org/10.1001/jama.2020.3151.

(5) Pang, J.; Wang, M. X.; Ang, I. Y. H.; Tan, S. H. X.; Lewis, R. F.; Chen, J. I.-P.; Gutierrez, R. A.; Gwee, S. X. W.; Chua, P. E. Y.; Yang, Q.; Ng, X. Y.; Yap, R. K. S.; Tan, H. Y.; Teo, Y. Y.; Tan, C. C.; Cook, A. R.; Yap, J. C.-H.; Hsu, L. Y. Potential Rapid Diagnostics, Vaccine and Therapeutics for 2019 Novel Coronavirus (2019-NCoV): A Systematic Review. J. Clin. Med. 2020, 9 (3), 623. https://doi.org/10.3390/jcm9030623.

(6) Yu, F.; Yan, L.; Wang, N.; Yang, S.; Wang, L.; Tang, Y.; Gao, G.; Wang, S.; Ma, C.; Xie, R.; Wang, F.; Tan, C.; Zhu, L.; Guo, Y.; Zhang, F.; Ditan Hospital, B.; Fujie Zhang, P. Quantitative Detection and Viral Load Analysis of SARS-CoV-2 in Infected Patients. 2020. https://doi.org/10.1093/cid/ciaa345/5812997.

(7) Salehi, S.; Abedi, A.; Balakrishnan, S.; Gholamrezanezhad, A. Coronavirus Disease 2019 (COVID-19): A Systematic Review of Imaging Findings in 919 Patients. Am. J. Roentgenol. 2020, 1-7. https://doi.org/10.2214/AJR.20.23034.

(8) Che, X. Y.; Qiu, L. W.; Pan, Y. X.; Wen, K.; Hao, W.; Zhang, L. Y.; Wang, Y. Di; Liao, Z. Y.; Hua, X.; Cheng, V. C. C.; Yuen, K. Y. Sensitive and Specific Monoclonal Antibody-Based Capture Enzyme Immunoassay for Detection of Nucleocapsid Antigen in Sera from Patients with Severe Acute Respiratory Syndrome. J. Clin. Microbiol. 2004, 42 (6), 2629-2635. https://doi.org/10.1128/JCM.42.6.2629-2635.2004.

(9) Di, B.; Hao, W.; Gao, Y.; Wang, M.; Wang, Y. Di; Qiu, L. W.; Wen, K.; Zhou, D. H.; Wu, X. W.; Lu, E. J.; Liao, Z. Y.; Mei, Y. B.; Zheng, B. J.; Che, X. Y. Monoclonal Antibody-Based Antigen Capture Enzyme-Linked Immunosorbent Assay Reveals High Sensitivity of the Nucleocapsid Protein in Acute-Phase Sera of Severe Acute Respiratory Syndrome Patients. Clin. Diagn. Lab. Immunol. 2005, 12 (1), 135-140. https://doi.org/10.1128/CDLI.12.1.135-140.2005.

(10) Cassaniti, I.; Novazzi, F.; Giardina, F.; Salinaro, F.; Sachs, M.; Perlini, S.; Bruno, R.; Mojoli, F.; Baldanti, F. Performance of VivaDiag COVID-19 IgM/IgG Rapid Test Is Inadequate for Diagnosis of COVID-19 in Acute Patients Referring to Emergency Room Department. J. Med. Virol. 2020, No. April, 1-4. https://doi.org/10.1002/jmv.25800. 
(11) Sharfstein, J. M.; Becker, S. J.; Mello, M. M. Diagnostic Testing for the Novel Coronavirus. JAMA 2020. https://doi.org/10.1001/jama.2020.3864.

(12) Incardona, S.; Serra-Casas, E.; Champouillon, N.; Nsanzabana, C.; Cunningham, J.; González, I. J. Global Survey of Malaria Rapid Diagnostic Test (RDT) Sales, Procurement and Lot Verification Practices: Assessing the Use of the WHO-FIND Malaria RDT Evaluation Programme (2011-2014). Malar. J. 2017, 16 (1), 196. https://doi.org/10.1186/s12936-017-1850-8.

(13) Products - STANDARD Q COVID-19 Ag http://sdbiosensor.com/xe/product/7672 (accessed May 3, 2020).

(14) Zou, L.; Ruan, F.; Huang, M.; Liang, L.; Huang, H.; Hong, Z.; Yu, J.; Kang, M.; Song, Y.; Xia, J.; Guo, Q.; Song, T.; He, J.; Yen, H. L.; Peiris, M.; Wu, J. SARS-CoV-2 Viral Load in Upper Respiratory Specimens of Infected Patients. New England Journal of Medicine. Massachussetts Medical Society March 19, 2020, pp 1177-1179. https://doi.org/10.1056/NEJMc2001737.

FIND. SARS-CoV-2 Diagnostics Pipeline https://www.finddx.org/covid19/pipeline/?section=immunoassays\#diag_tab (accessed Jan 5, 2020).

(16) Normile, D. Singapore Claims First Use of Antibody Test to Track Coronavirus Infections. Science (80-. ). 2020. https://doi.org/10.1126/science.abb4942.

(17) Li, Z.; Yi, Y.; Luo, X.; Xiong, N.; Liu, Y.; Li, S.; Sun, R.; Wang, Y.; Hu, B.; Chen, W.; Zhang, Y.; Wang, J.; Huang, B.; Lin, Y.; Yang, J.; Cai, W.; Wang, X.; Cheng, J.; Chen, Z.; Sun, K.; Pan, W.; Zhan, Z.; Chen, L.; Ye, F. Development and Clinical Application of a Rapid IgM-IgG Combined Antibody Test for SARS-CoV-2 Infection Diagnosis. J. Med. Virol. 2020. https://doi.org/10.1002/jmv.25727.

(18) Long, Q.; Deng, H.; Chen, J.; Hu, J.; Liu, B.; Liao, P.; Lin, Y.; Yu, L.; Mo, Z.; Xu, Y.; Gong, F.; Wu, G.; Zhang, X.; Chen, Y.; Li, Z.; Wang, K.; Zhang, X.; Tian, W.; Niu, C.; Yang, Q.; Xiang, J.; Du, H.; Liu, H.; Lang, C.; Luo, X.; Wu, S.; Cui, X.; Zhou, Z.; Wang, J.; Xue, C.; Li, X.; Wang, L.; Tang, X.; Zhang, Y.; Qiu, J.; Liu, X.; Li, J.; Zhang, D.; Zhang, F.; Cai, X.; Wang, D.; Hu, Y.; Ren, J.; Tang, N.; Liu, P.; Li, Q.; Huang, A. Antibody Responses to SARS-CoV-2 in COVID-19 Patients: The Perspective Application of Serological Tests in Clinical Practice. medRxiv 2020, 2020.03.18.20038018. https://doi.org/10.1101/2020.03.18.20038018.

(19) Vashist, S. K. In Vitro Diagnostic Assays for COVID-19: Recent Advances and Emerging Trends. Diagnostics. MDPI AG April 1, 2020. https://doi.org/10.3390/diagnostics10040202.

(20) NanoComposix. Measuring the Performance of Lateral Flow Assays https://nanocomposix.com/pages/testing-methods-for-measuring-the-performance-of-lateralflow-assays (accessed Mar 5, 2020).

(21) Ritz, C.; Baty, F.; Streibig, J.; Gerhard, D. Dose-Response Analysis Using R. PLoS One 2015, 10 (12), e0146021. https://doi.org/10.1371/journal.pone.0146021.t001.

(22) Armbruster, D. A.; Pry, T. Limit of Blank, Limit of Detection and Limit of Quantitation. Clin. Biochem. Rev. 2008, 29 Suppl 1 (Suppl 1), S49-52.

(23) Tu, Y.-P.; Jennings, R.; Hart, B.; Cangelosi, G.; Wood, R.; Wehber, K.; Verma, P.; Vojta, D.; Berke, E. M. Patient-Collected Tongue, Nasal, and Mid-Turbinate Swabs for SARS-CoV-2 Yield Equivalent Sensitivity to Health Care Worker Collected Nasopharyngeal Swabs. medRxiv 2020, 2020.04.01.20050005. https://doi.org/10.1101/2020.04.01.20050005. 
(24) Chang, C. K.; Hou, M. H.; Chang, C. F.; Hsiao, C. D.; Huang, T. H. The SARS Coronavirus Nucleocapsid Protein - Forms and Functions. Antiviral Res. 2014, 103 (1), 39-50. https://doi.org/10.1016/j.antiviral.2013.12.009.

(25) Burbelo, P. D.; Riedo, F. X.; Morishima, C.; Rawlings, S.; Smith, D.; Das, S.; Strich, J. R.; Chertow, D. S.; Davey, R. T.; Cohen, J. I. Detection of Nucleocapsid Antibody to SARS-CoV-2 Is More Sensitive than Antibody to Spike Protein in COVID-19 Patients. medRxiv 2020, 2020.04.20.20071423. https://doi.org/10.1101/2020.04.20.20071423.

(26) Zhao, B.; Ni, C.; Gao, R.; Wang, Y.; Yang, L.; Wei, J.; Lv, T.; Liang, J.; Zhang, Q.; Xu, W.; Xie, Y.; Wang, X.; Yuan, Z.; Liang, J.; Zhang, R.; Lin, X. Recapitulation of SARS-CoV-2 Infection and Cholangiocyte Damage with Human Liver Organoids. bioRxiv 2020, 2020.03.16.990317. https://doi.org/10.1101/2020.03.16.990317.

(27) V'kovski, P.; Gultom, M.; Steiner, S.; Kelly, J.; Russeil, J.; Mangeat, B.; Cora, E.; Pezoldt, J.; Holwerda, M.; Kratzel, A.; Laloli, L.; Wider, M.; Portmann, J.; Tran, T.; Ebert, N.; Stalder, H.; Hartmann, R.; Gardeux, V.; Alpern, D.; Deplancke, B.; Thiel, V.; Dijkman, R. Disparate Temperature-Dependent Virus - Host Dynamics for SARS-CoV-2 and SARS-CoV in the Human Respiratory Epithelium. bioRxiv 2020, 2020.04.27.062315. https://doi.org/10.1101/2020.04.27.062315. 


\section{Supplemental Information}

Table S1 - Raw data for dose response curve shown in Figure 1 for the two different sources of recombinant $\mathrm{N}$ proteins evaluated.

\begin{tabular}{|c|c|c|}
\hline & \multicolumn{2}{|c|}{ LFA Intensity (arb) } \\
\hline $\begin{array}{l}\text { conc } \\
(\mathrm{ng} / \mathrm{mL})\end{array}$ & Genemedi & Genscript \\
\hline 0 & 400 & 420 \\
\hline 0.25 & 460 & 360 \\
\hline 0.5 & 550 & 450 \\
\hline 1 & 680 & 510 \\
\hline 2 & 1190 & 520 \\
\hline 5 & 2360 & 810 \\
\hline 10 & 4720 & 1250 \\
\hline 20 & 8000 & 2060 \\
\hline 0 & 460 & 520 \\
\hline 0.25 & 440 & 460 \\
\hline 0.5 & 630 & 480 \\
\hline 1 & 760 & 450 \\
\hline 2 & 1180 & 520 \\
\hline 5 & 2480 & 910 \\
\hline 10 & 4920 & 1390 \\
\hline 20 & 8230 & 1910 \\
\hline 0 & 420 & 490 \\
\hline 0.25 & 580 & 480 \\
\hline 0.5 & 730 & 540 \\
\hline 1 & 870 & 500 \\
\hline 2 & 1380 & 690 \\
\hline 5 & 2810 & 990 \\
\hline 10 & 5400 & 1280 \\
\hline 20 & 7880 & 2870 \\
\hline
\end{tabular}

